\title{
False-Positive Accumulation of Metaiodobenzylguanidine in a Case with Acute Intermittent Porphyria
}

\author{
Tomoko Masuda ${ }^{1}$, Rie Ota ${ }^{1}$, Takao Ando ${ }^{1}$, Naoto Maeda ${ }^{2}$, Yutaka Horie ${ }^{3}$, \\ Toshiro Yoshimura ${ }^{1}$, Masakatsu Motomura ${ }^{1}$ and Atsushi Kawakami ${ }^{1}$
}

\begin{abstract}
We report a 36-year-old woman presenting with hypertensive encephalopathy followed by bulbar palsy and quadriplegia. After an extensive screening for secondary causes of hypertension, the patient was suspected of having pheochromocytoma due to increased levels of catecholamines in the plasma and the urine, and positive ${ }^{131}$ I-metaiodobenzylguanidine (MIBG) accumulation in the gallbladder. However, MIBG accumulation was not reproducible without any tumors accompanying this accumulation in the gallbladder. A diagnosis of acute intermittent porphyria was finally confirmed based on the characteristic pictures, increased urinary excretion of porphobilinogen, and identification of a heterozygous missense mutation of R173W in the hydroxymethylbilane synthase gene. This case highlights a pitfall in utilizing MIBG to detect a source of excessive catecholamine and also suggests the importance of having a complete clinical history and extensive work-up of any possible differential diagnosis. We also review the potential mechanism by which falsepositive MIBG accumulation occurs.
\end{abstract}

Key words: acute intermittent porphyria, MIBG, pheochromocytoma, false-positive

(Intern Med 50: 1029-1032, 2011)

(DOI: 10.2169/internalmedicine.50.5096)

\section{Introduction}

Metaiodobenzylguanidine (MIBG) has been widely used as a clinical tool to detect and localize pheochromocytoma since MIBG selectively accumulates in cells derived of neuroectodermal origin, including pheochromocytoma (1). It has been shown that the specificity of MIBG accumulation, when used to localize clinical pheochromocytomas, is as high as $95-100 \%$ (2). Reflecting excessive secretion of catecholamines from tumors, clinical symptoms seen in patients with pheochromocytoma are headache, anxiety, weight loss, nausea, and paroxysmal hypertension.

Acute intermittent porphyria (AIP) is characterized by episodic acute attacks of abdominal pain, headache, paroxysmal hypertension, seizures, confusion and hallucinations. Acute porphyria attacks can be life-threatening, since the motor polyneuropathy occasionally progresses to respiratory failure requiring a mechanical ventilator. Patients suffering from AIP, however, can be totally asymptomatic during the remission periods. This is because AIP is caused by reduced enzyme activity of hydroxymethylbilane synthase involving the heme biosynthesis, and excessive accumulation of neurotoxic heme precursors is only seen during and shortly after the attacks.

As described above, there is an overlap between such clinical symptoms of AIP and those of pheochromocytoma as paroxysmal hypertension and headache, and thus patients with AIP can be misdiagnosed with pheochromocytoma or vice versa. Here, we report a case of AIP with a confusing finding of false-positive MIBG accumulation suggesting pheochromocytoma. We also review the putative mechanisms involved in the false-positive MIBG accumulation.

\footnotetext{
${ }^{1}$ The First Department of Medicine, Nagasaki University Graduate School of Biomedical Sciences, Japan, ${ }^{2}$ The Second Department of Internal Medicine, Tottori University Faculty of Medicine, Japan and ${ }^{3}$ Department of Gastroenterology, Shimaneken Saiseikai-Gotsu General Hospital, Japan

Received for publication January 1, 2011; Accepted for publication January 12, 2011

Correspondence to Dr. Takao Ando, takaoando@gmail.com
} 
A

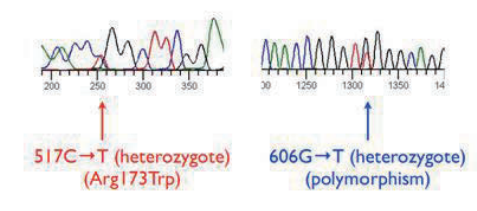

B

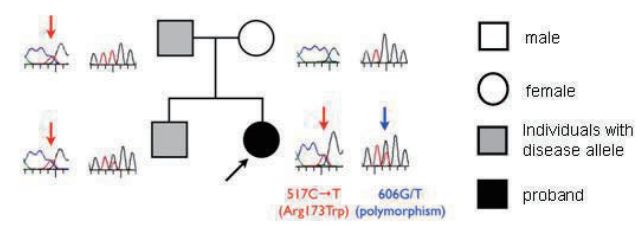

Figure 1. Identification of a missense mutation in hydroxymethylbilane synthase in the patient and her family members. (A) Genomic DNA taken from peripheral blood mononuclear cells were PCR-amplified with specific primers for the hydroxymethylbilane synthase gene and the PCR products were directly sequenced as described previously (20). A missense mutation of $\mathrm{R} 173 \mathrm{~W}$ is indicated by a red arrow and polymorphism of 606G to $\mathrm{T}$ is indicated by the blue arrow. (B) Genetic testing of the family members identified the disease allele in the father and sibling of the proband. The proband is indicated by the black arrow.

\section{Case Report}

A 36-year-old woman was admitted to the regional hospital in our area because of acute onset of visual field loss caused by hypertensive encephalopathy. While her visual field loss gradually improved by vigorous anti-hypertensive treatment, she developed progressive quadriplegia and bulbar paralysis and was therefore transferred to our university hospital. Her past medical history was unremarkable except for frequent episodes of headaches, nausea, vomiting, and generalized body pain associated with paroxysmal hypertension during the premenstrual period for more than ten years.

On physical examination, she was dehydrated and urinating port wine-colored urine. Additionally, there was apparent bulbar paralysis and quadriplegia associated with a loss of deep tendon reflexes. Since the patient was intubated and under sedation, we were not able to ask her whether she had sensation in her limbs. A blood test showed moderate anemia and the anti-ganglioside antibodies studied were negative. Cerebrospinal fluid analysis was normal. Nerve conduction study in the median, ulnar, peroneal, and tibial nerve showed normal conduction velocity with a low amplitude (amplitude and velocity was $0.79 \mathrm{mV}$ and $44.0 \mathrm{~m} / \mathrm{sec}$ in the median nerve and $1.5 \mathrm{mV}$ and $46.4 \mathrm{~m} / \mathrm{sec}$ in the ulnar) indicating axonal motor polyneuropathy while there was no involvement of sensory nerves. From the clinical picture of the patient, we strongly suspected an attack of acute porphyria. This was supported by a markedly elevated urinary concentration of porphobilinogen (134.2 mg/day; reference range less than $2 \mathrm{mg} /$ day). Other urinary porphy- rins were also increased; 5-aminolevulinic acid $(22.3 \mathrm{mg} / \mathrm{L}$; reference range $<5 \mathrm{mg} / \mathrm{L})$, uroporphyrin $(1,820 \mu \mathrm{g} / \mathrm{gCr}$; reference range $<36 \mu \mathrm{g} / \mathrm{gCr})$ and coproporphyrin $(8,540 \mu \mathrm{g} /$ $\mathrm{gCr}$; reference range $170 \mu \mathrm{g} / \mathrm{gCr}$ ). After obtaining written informed consent from the patient and her family and approval from the ethical committee at Tottori University Hospital (Tottori, Japan), we were also able to detect a heterozygous mutation in the hydroxymethylbilane synthase gene ( $\mathrm{R}$ $173 \mathrm{~W}$ caused by $517 \mathrm{C}$ to $\mathrm{T}$ in exon 10 ), which confirmed the diagnosis of AIP (Fig. 1A). There was an additional polymorphism of $606 \mathrm{G}$ to $\mathrm{T}$ (Fig. 1A), and genetic testing of her family members revealed that the disease allele was paternally inherited (Fig. 1B). The patient was treated with intravenous hyperalimentation, cimetidine, and chlorpromazine, and the neurological signs and symptoms gradually improved.

Before being transferred to our hospital, the patient was extensively investigated for the possibility of secondary hypertension as an etiology of hypertensive encephalopathy. Plasma levels of catecholamines and urinary levels of catecholamine metabolites were found to be elevated: plasma adrenaline $(242 \mathrm{pg} / \mathrm{mL}$; reference range $<100 \mathrm{pg} /$ $\mathrm{mL})$, noradrenaline $(2,583 \mathrm{pg} / \mathrm{mL}$; reference range 100 to $450 \mathrm{pg} / \mathrm{mL})$, dopamine $(231 \mathrm{pg} / \mathrm{mL}$; reference range $<20 \mathrm{pg} /$ $\mathrm{mL})$ and urinary metanephrine $(0.22 \mathrm{mg} /$ day; reference range 0.04 to $0.19 \mathrm{mg} / \mathrm{day})$, normetanephrine $(1.37 \mathrm{mg} / \mathrm{day}$; reference range 0.09 to $0.33 \mathrm{mg} /$ day $)$, VMA $(9.0 \mathrm{mg} /$ day; reference range 1.5 to $4.3 \mathrm{mg} /$ day). Therefore, a diagnosis of pheochromocytoma was considered and ${ }^{131} \mathrm{I}-\mathrm{MIBG}$ scintigraphy showed positive accumulation in the gallbladder (Fig. 2A, 2B). However, we were not able to find any tumorous lesions in or near the gallbladder by ultrasound (Fig. 2C). ${ }^{123}$ I-MIBG accumulation studied 14 days after the ${ }^{131}$ I-MIBG study showed no accumulation of MIBG in the gallbladder or adrenal gland (Fig. 2D), and there was a gradual decrease of urinary metanephrine.

\section{Discussion}

The case reported here was first presented with hypertensive encephalopathy caused by paroxysmal hypertension subsequently complicated with bulbar palsy and quadriplegia as a result of axonal motor polyneuropathy. The diagnosis of AIP was made based on the characteristic clinical presentation, increased urinary excretion of porphobilinogen, and genetic testing. It has been shown that mutations were widely distributed within the gene coding hydroxymethylbilane synthase, and the mutation identified in the present patient was also observed in Caucasians as well as in other unrelated Japanese patients with AIP (3-5). It has also been shown that the mutation is the substitution of an essential arginine to tryptophan in the active site of the enzyme, decreasing catalytic activity $(<1 \%)(6)$. The patient could have been incorrectly diagnosed with pheochromocytoma based on her paroxysmal hypertension and false-positive accumulation of ${ }^{131}$ I-MIBG. A misdiagnosis of this kind can easily occur in 
A

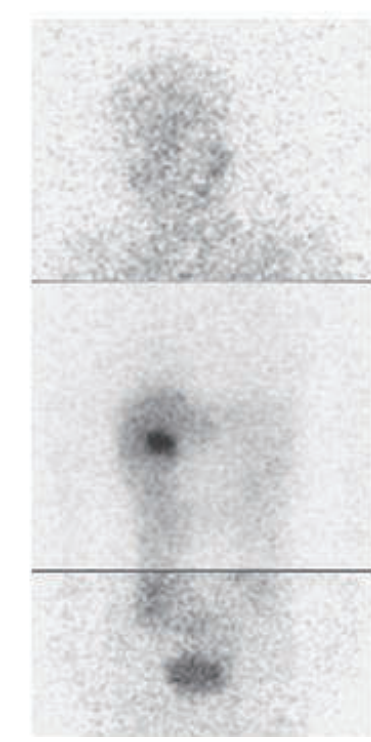

B

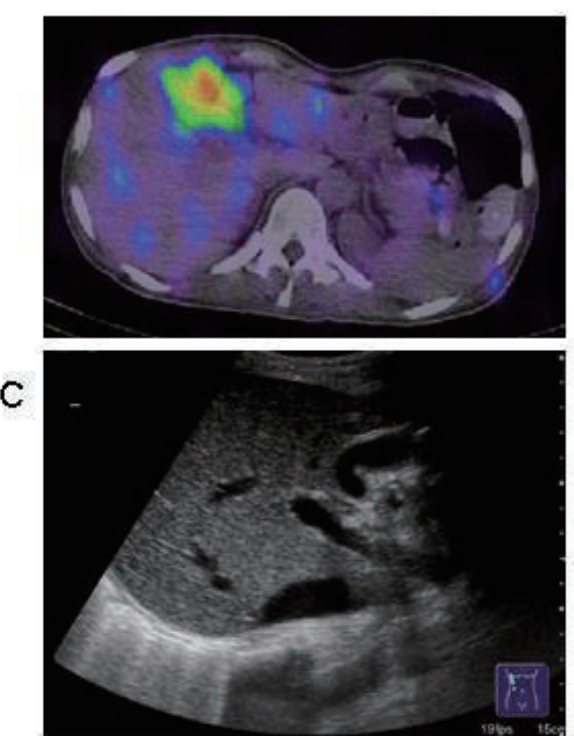

D

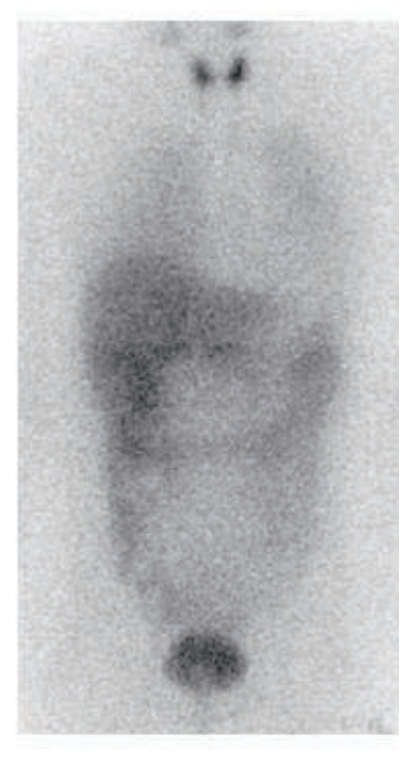

Figure 2. False-positive accumulation of MIBG in the gallbladder. There is clear accumulation of ${ }^{131}$ I-MIBG in the right upper abdomen (A), which corresponds to the gallbladder, as shown in SPECT (B). There was no detectable tumor in or near the gallbladder on ultrasound (C). ${ }^{123}$ I-MIBG scintigraphy taken 14 days after ${ }^{131}$ I-MIBG showed no abnormal accumulation (D).

Japan, where AIP is extremely rare [up to 198 cases of AIP have been diagnosed as of 2009 (7)]. In addition, the clinical presentation of pheochromocytoma can vary greatly and mimic signs and symptoms seen in many other disorders (2).

It has been shown that acute porphyria attacks are commonly seen in females, although very rarely before puberty and after menopause, with a peak occurrence within the third decade $(8,9)$. Most patients have one or two attacks and then fully recover without a recurrence for the rest of their lives, but less than 10\% develop recurrent acute attacks. During the acute attacks, reflecting augmented sympathetic activity, tachycardia, excessive sweating, and hypertension are commonly present (10). As shown herein, increased catecholamine production can be detected and may possibly suggest an incorrect diagnosis. When an acute attack of porphyria is suspected, it is essential to obtain a complete clinical history, perform an extensive work up of any possible differentiating diagnosis, and determine the urinary concentration of porphobilinogen (11).

MIBG is a norepinephrine analogue which is taken up by neuroendocrine cells through an active mechanism and stored in the neurosecretory granules. This leads to a specific concentration of the molecule in the neuroendocrine cells (1). Only limited reports of false-positive uptake in other lesions have been published. One major cause of falsepositive findings is urinary tract retention, since the reagent is excreted in the urine (12). Other rare false-positive MIBG accumulations have been reported in the adrenal gland with adenoma (13), carcinoma (14) or metastatic choriocarcinoma, and adenomatous polyp of the cecum, infantile myofibromatosis (15), pancreatoblastoma (15), acute focal pyelonephritis, hepatic hemangioma, hepatocellular carcinoma (16), and juvenile capillary hemangioma (17). The non-specific accumulation of MIBG is thought to be mediated by a passive uptake and diffusion. It has been shown that non-specific uptake tends to disappear more rapidly than specific uptake (15). An augmented blood flow and enhanced diffusion of MIBG within the tumor could be putative mechanisms of false-positive MIBG uptake and accumulation in non-neuroendocrine tumors $(15,18)$.

There was false-positive accumulation of MIBG in the gallbladder in the case with AIP reported here. To our knowledge, there is no report of a similar false-positive MIBG accumulation. The accumulation of MIBG in the gallbladder can be considered a characteristic finding in patients with AIP. Since the liver is one of the major organs involving heme biosynthesis, which is partially defective in AIP, MIBG clearance might be disturbed during acute attacks of AIP. It is not known whether MIBG accumulates in any specific organ in patients with AIP. False-positive MIBG accumulation in the normal adrenal gland in a patient with AIP has in fact been reported (19). Thus, it seems unlikely that MIBG tends to accumulate in the gallbladder in AIP although there have been no studies confirming this. The other possibility is that the critical condition of the patient reported herein might have influenced the blood flow of the gallbladder, as the patient was severely ill and receiving intravenous hyperalimentation when ${ }^{131} \mathrm{I}-\mathrm{MIBG}$ accumulation was studied. We were not able to find any studies on the blood flow of the gallbladder in patients with intravenous hyperalimentation, in which the gallbladder is known to dilate. However, the increased blood flow in the gallbladder would not have been the cause of false-positive MIBG accu- 
mulation since the patient was still under intravenous hyperalimentation when ${ }^{123}$ I-MIBG scintigraphy was studied.

In conclusion, an acute attack of porphyria should be considered when female patients of reproductive age present with characteristic episodes of neurovisceral symptoms. However, the present patient could have been incorrectly diagnosed with pheochromocytoma based on MIBG accumulation in the gallbladder. The possibility of a false-positive MIBG accumulation should always be taken into consideration when using MIBG scintigraphy to detect a catecholamine-secreting tumor, or the result of the MIBG can be misleading.

Author's disclosure of potential Conflicts of Interest (COI). Astushi Kawakami: Research funding, Ministry of Health, Labor and Welfare of Japan.

\section{Acknowledgement}

The first two authors (T.M., and R.O.) contributed equally to this report.

\section{References}

1. Team TMR. Meta-[radioiodinated]iodobenzylguanidine. Bethesda: National Center for Biotechnology Information, US, 2007.

2. Lenders JW, Eisenhofer G, Mannelli M, Pacak K. Phaeochromocytoma. Lancet 366: 665-675, 2005.

3. Tomie Y, Horie Y, Tajima F, et al. Mutation in the exon 10 (R173 W) of the hydroxymethylbilane synthase gene in two unrelated Japanese families with acute intermittent porphyria. Res Commun Mol Pathol Pharmacol 99: 5-15, 1998.

4. Greene-Davis ST, Neumann PE, Mann OE, et al. Detection of a R $173 \mathrm{~W}$ mutation in the porphobilinogen deaminase gene in the Nova Scotian "foreign Protestant" population with acute intermittent porphyria: a founder effect. Clin Biochem 30: 607-612, 1997.

5. To-Figueras J, Badenas C, Carrera $\mathrm{C}$, et al. Genetic and biochemical characterization of 16 acute intermittent porphyria cases with a high prevalence of the R173W mutation. J Inherit Metab Dis 29: 580-585, 2006

6. Mustajoki S, Laine M, Lahtela M, Mustajoki P, Peltonen L, Kauppinen R. Acute intermittent porphyria: expression of mutant and wild-type porphobilinogen deaminase in COS-1 cells. Mol Med 6: 670-679, 2000.

7. Kondo M, Kondo T. Porphyria in Japan: The past, present, and future. Porphyrins 18: 1-6, 2010.

8. Bylesjo I, Wikberg A, Andersson C. Clinical aspects of acute intermittent porphyria in northern Sweden: a population-based study. Scand J Clin Lab Invest 69: 612-618, 2009.

9. Andersson C, Innala E, Backstrom T. Acute intermittent porphyria in women: clinical expression, use and experience of exogenous sex hormones. A population-based study in northern Sweden. J Intern Med 254: 176-183, 2003.

10. Hift RJ, Meissner PN. An analysis of 112 acute porphyric attacks in Cape Town, South Africa: Evidence that acute intermittent porphyria and variegate porphyria differ in susceptibility and severity. Medicine (Baltimore) 84: 48-60, 2005.

11. Puy H, Gouya L, Deybach JC. Porphyrias. Lancet 375: 924-937.

12. Kao PF, Tzen KY, Huang MJ, You DL. Obstructive hydronephrosis with I-131 MIBG accumulation mimicking huge pheochromocytoma: a diagnostic pitfall found with Tc-99m MDP imaging. Clin Nucl Med 21: 994-995, 1996.

13. Sone H, Okuda Y, Nakamura Y, et al. Radioiodinated metaiodobenzylguanidine scintigraphy for pheochromocytoma. A falsepositive case of adrenocortical adenoma and literature review. Horm Res 46: 138-142, 1996.

14. Rainis T, Ben-Haim S, Dickstein G. False positive metaiodobenzylguanidine scan in a patient with a huge adrenocortical carcinoma. J Clin Endocrinol Metab 85: 5-7, 2000.

15. Leung A, Shapiro B, Hattner R, et al. Specificity of radioiodinated MIBG for neural crest tumors in childhood. J Nucl Med 38: 13521357, 1997.

16. Inoue $Y$, Akahane M, Kitazawa $T$, et al. False positive uptake of metaiodobenzylguanidine in hepatocellular carcinoma. Br J Radiol 75: 548-551, 2002.

17. Frappaz D, Giammarile F, Thiesse P, et al. False positive MIBG scan. Med Pediatr Oncol 29: 589-592, 1997.

18. Stewart RE, Grossman DM, Shulkin BL, Shapiro B. Iodine-131 metaiodobenzylguanidine uptake in infantile myofibromatosis. Clin Nucl Med 14: 344-346, 1989.

19. Stewart MF, Croft J, Reed P, New JP. Acute intermittent porphyria and phaeochromocytoma: shared features. J Clin Pathol 60: 935936, 2007.

20. Maeda N, Horie Y, Sasaki Y, et al. A splicing mutation in the hydroxymethylbilane synthase gene in a Japanese family with acute intermittent porphyria. Clin Biochem 32: 411-417, 1999.

\footnotetext{
(C) 2011 The Japanese Society of Internal Medicine http://www.naika.or.jp/imindex.html
} 